University of Wisconsin - Madison

MADPH-00-1180

May 2000

\title{
Neutrino Mixing Schemes
}

\author{
V. Barger ${ }^{a}$ and K. Whisnant ${ }^{b}$ \\ ${ }^{a}$ Department of Physics, University of Wisconsin, Madison, WI 53706 \\ ${ }^{b}$ Department of Physics and Astronomy, Iowa State University, Ames, IA 50011
}

\section{Introduction}

A revolution in our understanding of the neutrino sector is underway, driven by observations that are interpreted in terms of changes in neutrino flavors as they propagate. Since neutrino oscillations occur only if neutrinos are massive, these phenomena indicate physics beyond the Standard Model. With the present evidence for oscillations from atmospheric, solar and accelerator data, we are already able to begin to make strong inferences about the mass spectrum and mixings of neutrinos. Theoretical efforts to achieve a synthesis have produced a variety of models with differing testable consequences. A combination of particle physics, nuclear physics and astrophysics is needed for a full determination of the fundamental properties of neutrinos. This article reviews what has been achieved thus far and the future prospects for understanding the nature of neutrino masses and mixing.

\section{Two-Neutrino Analyses}

In a model with two neutrinos, the probability for a given neutrino flavor $\nu_{\alpha}$ oscillating into $\nu_{\beta}$ in a vacuum is

$$
P\left(\nu_{\alpha} \rightarrow \nu_{\beta}\right)=\sin ^{2} 2 \theta \sin ^{2} 1.27 \frac{\delta m^{2} L}{E},
$$

where $\theta$ is the two-neutrino mixing angle, $\delta m^{2}$ is the mass-squared difference of the two mass eigenstates in $\mathrm{eV}^{2}, L$ is the distance from the neutrino source to the detector in kilometers, and $E$ is the neutrino energy in $\mathrm{GeV}$.

To appear in "Current Aspects of Neutrino Physic", ed. by D. Caldwell (SpringerVerlag, Hamburg, 2000) 


\subsection{Atmospheric neutrinos}

The atmospheric neutrino experiments determine the ratios

$$
\begin{aligned}
& \frac{N_{\mu}}{N_{\mu}^{0}}=\alpha\left[\left\langle P\left(\nu_{\mu} \rightarrow \nu_{\mu}\right)\right\rangle+r\left\langle P\left(\nu_{e} \rightarrow \nu_{\mu}\right\rangle\right],\right. \\
& \frac{N_{e}}{N_{e}^{0}}=\alpha\left[\left\langleP\left(\nu_{e} \rightarrow \nu_{e}\right\rangle+r^{-1}\left\langle P\left(\nu_{\mu} \rightarrow \nu_{e}\right\rangle\right],\right.\right.
\end{aligned}
$$

where $N_{e}^{0}$ and $N_{\mu}^{0}$ are the expected numbers of atmospheric $e$ and $\mu$ events,

respectively, in the absence of oscillations, $r \equiv \frac{N_{e}^{0}}{N_{\mu}^{0}},\langle\rangle$ indicates an average over the neutrino spectrum, and $\alpha$ is an overall neutrino flux normalization. Atmospheric neutrino data have generally indicated that the expected number of muons detected is suppressed relative to the expected number of electrons [1]; this suppression can be explained via neutrino oscillations [2].

The atmospheric data also indicate that $N_{e} / N_{e}^{0}$ is relatively flat with zenith angle, while $N_{\mu} / N_{\mu}^{0}$ decreases with increasing zenith angle (i.e., with longer oscillation distance). Assuming $\nu_{\mu} \rightarrow \nu_{\tau}$ oscillations, the favored twoneutrino parameters are [3]

$$
\begin{aligned}
\delta m^{2} & =3.5 \times 10^{-3} \mathrm{eV}^{2} \quad\left(1.5-7 \times 10^{-3} \mathrm{eV}^{2}\right), \\
\sin ^{2} 2 \theta & =1.00 \quad(0.80-1.00) ;
\end{aligned}
$$

the $90 \%$ C.L. allowed ranges are given in parentheses. The absolute normalization of the electron data indicates $\alpha \simeq 1.18$, which is within the theoretical uncertainties [ $[$. The flatness versus $L$ of the electron data implies that simple $\nu_{\mu} \rightarrow \nu_{e}$ oscillations are strongly disfavored. Large amplitude $\left(\sin ^{2} 2 \theta>0.2\right)$ $\nu_{\mu} \rightarrow \nu_{e}$ oscillations are also excluded by the CHOOZ reactor data [5] for $\delta m_{\text {atm }}^{2} \gtrsim 10^{-3} \mathrm{eV}^{2}$.

It is also possible that atmospheric $\nu_{\mu}$ are oscillating into sterile neutrinos. However, measurements of the upgoing zenith angle distribution and $\pi^{0}$ production disfavor this possibility [6, 7].

\subsection{Solar neutrinos}

For the ${ }^{37} \mathrm{Cl}[8]$ and ${ }^{71} \mathrm{Ga}[9]$ experiments, the expected number of neutrino events is

$$
N=\int \sigma P\left(\nu_{e} \rightarrow \nu_{e}\right)\left(\beta \phi_{\mathrm{B}}+\phi_{\text {non-B }}\right) d E,
$$

where we allow an arbitrary normalization factor $\beta$ for the ${ }^{8} \mathrm{~B}$ neutrino flux since its normalization is not certain. For the Kamiokande [10] and SuperKamiokande [11] experiments the interaction is $\nu e \rightarrow \nu e$ and the outgoing 
electron energy is measured. The number of events per unit of electron energy is then

$$
\begin{gathered}
\frac{d N}{d E_{e}}=\beta \int\left\{\frac{d \sigma_{C C}}{d E_{e}^{\prime}} P\left(\nu_{e} \rightarrow \nu_{e}\right)+\frac{d \sigma_{N C}}{d E_{e}^{\prime}}\left[1-P\left(\nu_{e} \rightarrow \nu_{e}\right)\right]\right\} \\
\times G\left(E_{e}^{\prime}, E_{e}\right) \phi_{\mathrm{B}} d E_{\nu} d E_{e}^{\prime}
\end{gathered}
$$

where $d \sigma_{C C} / d E_{e}^{\prime}\left(d \sigma_{N C} / d E_{e}^{\prime}\right)$ is the charged-current (neutral-current) differential cross section for an incident neutrino energy $E_{\nu}$ and $G\left(E_{e}^{\prime}, E_{e}\right)$ is the probability that an electron of energy $E_{e}^{\prime}$ is measured as having energy $E_{e}$. The neutrino fluxes are taken from the standard solar model (SSM) [12]. If $\nu_{e}$ oscillates into a sterile neutrino, $\sigma_{N C}=0$.

For two-neutrino vacuum oscillations (VO) of $\nu_{e}$ into either $\nu_{\mu}$ or $\nu_{\tau}$ [13], the solar data indicate 114,15

$$
\begin{aligned}
\delta m^{2} & =7.5 \times 10^{-11} \mathrm{eV}^{2}, \\
\sin ^{2} 2 \theta & =0.91,
\end{aligned}
$$

although there are also regions near $\delta m^{2}=2.5 \times 10^{-10}, 4.4 \times 10^{-10}$, and $6.4 \times$ $10^{-10} \mathrm{eV}^{2}$ that also give acceptable fits.

For two-neutrino MSW oscillations [16] of $\nu_{e}$ into either $\nu_{\mu}$ or $\nu_{\tau}$, there are three possible solution regions [15]. The small-angle MSW (SAM) solution is

$$
\begin{aligned}
\delta m^{2} & =7.5 \times 10^{-6} \mathrm{eV}^{2}, \\
\sin ^{2} 2 \theta & =0.01,
\end{aligned}
$$

and the large-angle MSW (LAM) solution is

$$
\begin{aligned}
\delta m^{2} & \sim 10^{-5} \mathrm{eV}^{2}, \\
\sin ^{2} 2 \theta & =0.6-0.9 .
\end{aligned}
$$

There is also a low $\delta m^{2}$ MSW (LOW) solution

$$
\begin{aligned}
\delta m^{2} & \sim 10^{-7} \mathrm{eV}^{2}, \\
\sin ^{2} 2 \theta & =0.6-0.9
\end{aligned}
$$

although it is less favored. Note that all solutions except for small-angle MSW have large mixing in the solar sector.

Two-neutrino oscillations of $\nu_{e}$ into a sterile neutrino are somewhat disfavored because sterile neutrinos do not have a NC interaction, which tends to make oscillation predictions for ${ }^{37} \mathrm{Cl}$ and SuperK similar, contrary to experimental evidence. Future measurements of NC interactions in the SNO detector [17] will provide a more thorough test for oscillations of solar $\nu_{e}$ into sterile neutrinos.

SuperK and SNO will also provide an improved measurement of the ${ }^{8} \mathrm{~B}$ neutrino spectrum in the near future, which should help distinguish the various solar scenarios. The Borexino 18 and ICARUS [19] experiments will provide a measurement of the ${ }^{7} \mathrm{Be}$ neutrinos, and could detect the strong seasonal dependence that exists for ${ }^{7} \mathrm{Be}$ neutrinos in many VO models [20]. 


\subsection{LSND}

There are also indications of neutrino oscillations from the LSND accelerator experiment [21]. Their data suggest $\nu_{\mu} \rightarrow \nu_{e}$ oscillations with two-neutrino parameters,

$$
0.3 \mathrm{eV}^{2} \leq \delta m^{2}=\frac{0.03 \mathrm{eV}^{2}}{\left(\sin ^{2} 2 \theta\right)^{0.7}} \leq 2.0 \mathrm{eV}^{2} .
$$

Values of $\delta m^{2}$ above $10 \mathrm{eV}^{2}$ are also allowed for $\sin ^{2} 2 \theta \simeq 0.0025$ 22. The future MiniBooNE experiment [23] is expected to either confirm or refute the LSND result.

\section{Global Analyses}

A complete description of all neutrino data requires a model that can explain all of the oscillation phenomena simultaneously. Since each of the three types of oscillation evidence (atmospheric, solar, LSND) requires a distinct $\delta m^{2}$ scale, and since $N$ neutrinos have only $N-1$ independent mass-squared differences, four neutrinos are needed to completely explain all of the data. If one of the types of neutrino data is ignored, then it is in principle possible to explain the remaining data with a three-neutrino model. Because the LSND results have yet to be confirmed by another experiment, three-neutrino models are generally used in the context of describing the atmospheric and solar data.

\subsection{Three neutrinos}

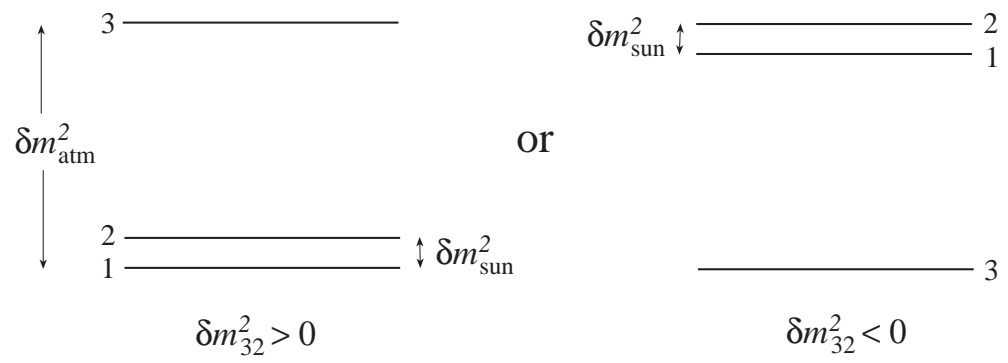

Fig. 1. Two possible patterns of neutrino masses that can explain the atmospheric and solar anomalies.

If the atmospheric and solar data are to be described by a three-neutrino model, there are two distinct mass-squared difference scales [compare Eq. (4) with Eqs. (8), (10), (12) and (14)]. The two possible patterns of three-neutrino masses are illustrated in Fig. 1. We assume without loss of generality that 
$\left|m_{1}\right|<\left|m_{2}\right|<\left|m_{3}\right|$, and that $\delta m_{21}^{2} \equiv \delta m_{\text {sun }}^{2}$ and $\delta m_{31}^{2} \simeq \delta m_{32}^{2} \equiv \delta m_{\text {atm }}^{2} \gg$ $\delta m_{\text {sun }}^{2}$. Then the off-diagonal vacuum oscillation probabilities in a threeneutrino model are 24]

$$
\begin{aligned}
P\left(\nu_{e} \rightarrow \nu_{\mu}\right)= & 4\left|U_{e 3} U_{\mu 3}^{*}\right|^{2} \sin ^{2} \Delta_{\mathrm{atm}}-4 \mathcal{R} e\left\{U_{e 1} U_{e 2}^{*} U_{\mu 1}^{*} U_{\mu 2}\right\} \sin ^{2} \Delta_{\text {sun }} \\
& -2 J \sin 2 \Delta_{\text {sun }}, \\
P\left(\nu_{e} \rightarrow \nu_{\tau}\right)= & 4\left|U_{e 3} U_{\tau 2}^{*}\right|^{2} \sin ^{2} \Delta_{\mathrm{atm}}-4 \operatorname{Re}\left\{U_{e 1} U_{e 2}^{*} U_{\tau 1}^{*} U_{\tau 2}\right\} \sin ^{2} \Delta_{\text {sun }} \\
& +2 J \sin 2 \Delta_{\text {sun }}, \\
P\left(\nu_{\mu} \rightarrow \nu_{\tau}\right)= & 4\left|U_{\mu 3} U_{\tau 3}^{*}\right|^{2} \sin ^{2} \Delta_{\mathrm{atm}}-4 \operatorname{Re}\left\{U_{\mu 1} U_{\mu 2}^{*} U_{\tau 1}^{*} U_{\tau 2}\right\} \sin ^{2} \Delta_{\text {sun }} \\
& -2 J \sin 2 \Delta_{\text {sun }},
\end{aligned}
$$

where $U$ is the neutrino mixing matrix (in the basis where the chargedlepton mass matrix is diagonal), $\Delta_{j} \equiv 1.27\left(\delta m_{j}^{2} / \mathrm{eV}^{2}\right)(L / \mathrm{km})(E / \mathrm{GeV})$ and $J=\operatorname{Im}\left\{U_{e 2} U_{e 3}^{*} U_{\mu 2}^{*} U_{\mu 3}\right\}$ is the CP-violating invariant [25]. For a discussion of $\mathrm{CP}$ violating effects in neutrino oscillations see Refs. [26, 27,28

The matrix elements $U_{\alpha j}$ are the mixing between flavor $(\alpha=e, \mu, \tau)$ and the mass $(j=1,2,3)$ eigenstates. The $\mathrm{CP}$-odd term changes sign under reversal of the oscillating flavors, or if neutrinos are replaced by anti-neutrinos. For either Dirac or Majorana neutrinos we choose the following parametrization for $U$ to describe neutrino oscillations

$$
U=\left(\begin{array}{ccc}
c_{13} c_{12} & c_{13} s_{12} & s_{13} e^{-i \delta} \\
-c_{23} s_{12}-s_{13} s_{23} c_{12} e^{i \delta} & c_{23} c_{12}-s_{13} s_{23} s_{12} e^{i \delta} & c_{13} s_{23} \\
s_{23} s_{12}-s_{13} c_{23} c_{12} e^{i \delta} & -s_{23} c_{12}-s_{13} c_{23} s_{12} e^{i \delta} & c_{13} c_{23}
\end{array}\right),
$$

where $c_{j k} \equiv \cos \theta_{j k}$ and $s_{j k} \equiv \sin \theta_{j k}$. For Majorana neutrinos, $U$ contains two further phase factors, but these do not enter into oscillation phenomena.

For the oscillation of neutrinos in atmospheric and long-baseline experiments with $L / E \gtrsim 10^{2} \mathrm{~km} / \mathrm{GeV}$, the $\Delta_{\text {sun }}$ terms are negligible and the relevant vacuum oscillation probabilities are

$$
\begin{aligned}
& P\left(\nu_{e} \rightarrow \nu_{\mu}\right)=s_{23}^{2} \sin ^{2} 2 \theta_{13} \sin ^{2} \Delta_{\mathrm{atm}}, \\
& P\left(\nu_{e} \rightarrow \nu_{\tau}\right)=c_{23}^{2} \sin ^{2} 2 \theta_{13} \sin ^{2} \Delta_{\mathrm{atm}}, \\
& P\left(\nu_{\mu} \rightarrow \nu_{\tau}\right)=c_{13}^{4} \sin ^{2} 2 \theta_{23} \sin ^{2} \Delta_{\mathrm{atm}} .
\end{aligned}
$$

The diagonal oscillation probabilities for a given neutrino species can be found by subtracting all the off-diagonal probabilities involving that species from unity.

For neutrinos from the sun, $L / E \sim 10^{10} \mathrm{~km} / \mathrm{GeV}$, and the $\sin ^{2} \Delta_{\text {atm }}$ terms oscillate very rapidly, averaging to $1 / 2$. Then the observable oscillation probability in a vacuum is

$$
P\left(\nu_{e} \rightarrow \nu_{e}\right)=1-\frac{1}{2} \sin ^{2} 2 \theta_{13}-c_{13}^{4} \sin ^{2} 2 \theta_{12} \sin ^{2} \Delta_{\text {sun }} .
$$

When $\theta_{13}=0$ (i.e., $U_{13}=0$ ), the oscillations of atmospheric and long-baseline neutrinos decouple from those of solar neutrinos: at the $\delta m_{\mathrm{atm}}^{2}$ scale, there 
are pure $\nu_{\mu} \rightarrow \nu_{\tau}$ oscillations with amplitude $\sin ^{2} 2 \theta_{23}$, with no admixture of $\nu_{e}$, and at the $\delta m_{\text {sun }}^{2}$ scale the $\nu_{e}$ oscillations are described by a simple twoneutrino formula with amplitude $\sin ^{2} 2 \theta_{12}$. Then the two-neutrino parameters for atmospheric and solar oscillations can be adopted directly in the threeneutrino case.

If $\theta_{13} \neq 0$, then $\nu_{e}$ will participate in atmospheric and long-baseline oscillations. As discussed earlier, pure $\nu_{\mu} \rightarrow \nu_{e}$ oscillations at the $\delta m_{\text {atm }}^{2}$ scale are strongly disfavored by the atmospheric data, but some small amount of $\nu_{\mu} \rightarrow \nu_{e}$ is still allowed. The CHOOZ reactor experiment measures $\bar{\nu}_{e}$ survival, and is sensitive to oscillations between $\bar{\nu}_{e}$ and $\bar{\nu}_{\mu}$ for $\delta m_{\mathrm{atm}}^{2}>$ $10^{-3} \mathrm{eV}^{2}$. The combined data from atmospheric experiments and $\mathrm{CHOOZ}$ favor $\sin \theta_{13}=0$ (i.e., pure $\nu_{\mu} \rightarrow \nu_{\tau}$ oscillations in the atmosphere) and suggest that $\sin \theta_{13}<0.3$ 24.

The solar data also allow solar neutrinos to mix maximally, or nearly maximally. If we require both atmospheric and solar oscillations to be maximal, there is a unique three-neutrino solution to the neutrino mixing matrix [29]. This "bimaximal" mixing corresponds to $\theta_{13}=0$ and $\left|\theta_{12}\right|=\left|\theta_{23}\right|=\pi / 4$, and is a special case of the decoupled solution for atmospheric and solar neutrinos. One interesting aspect of the bimaximal solution is that the solar $\nu_{e}$ oscillations are $50 \%$ into $\nu_{\mu}$ and $50 \%$ into $\nu_{\tau}$, although the flavor content of the $\nu_{e}$ oscillation is not observable in solar experiments. Further properties of the bimaximal and nearly bimaximal solutions and models that give rise to such solutions are discussed in Ref. 229].

\subsection{Four neutrinos}

As discussed earlier, four neutrinos are necessary to completely describe the atmospheric, solar and LSND results. A fourth light neutrino must be sterile since it is not observed in $Z$ decays [30]. There must be three separate masssquared scales which must satisfy the hierarchy $\delta m_{\text {sun }}^{2} \ll \delta m_{a t m}^{2} \ll \delta m_{L S N D}^{2}$. In the three-neutrino case the relation $\delta m_{\text {sun }}^{2} \ll \delta m_{\text {atm }}^{2}$ leads to only one fundamental type of mass structure, in which one heavier mass is separated from two lighter, nearly degenerate states (or vice versa). In the four-neutrino case, however, there are two distinct types of mass structures: one heavier mass separated from three lighter, nearly degenerate states, or vice versa (which we will refer to as the $1+3$ spectrum), or two nearly degenerate mass pairs separated from each other (the $2+2$ spectrum). In each case, the largest separation scale is determined by the LSND scale. It can be shown [31,32] that only the $2+2$ spectrum is completely consistent with the positive oscillation signals in the solar, atmospheric and LSND experiments, and the negative results from the BUGEY reactor [33] and CDHS accelerator [34] experiments. Therefore our discussions below assume the $2+2$ case, which is illustrated in Fig. 2. Here we will assume that the mass splitting of the heavier pair drives the atmospheric oscillations, the mass splitting of the lighter pair drives the solar oscillations, and the separation of the mass pairs drives 
the LSND oscillations. Sterile neutrinos are also of interest in explaining $r$ process nucleosynthesis via supernova explosions (see e.g. Ref. [22]).

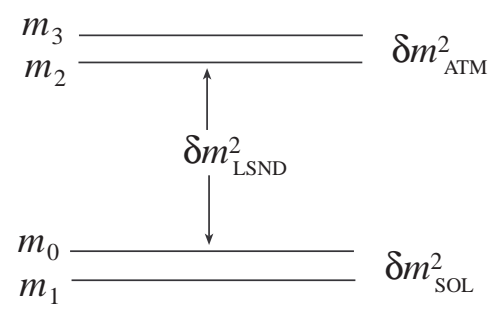

Fig. 2. Required mass pattern of two separated pairs to account for the LSND, atmospheric, and solar data; the locations of $\delta m_{\mathrm{ATM}}^{2}$ and $\delta m_{\mathrm{SUN}}^{2}$ may be interchanged.

The vacuum neutrino flavor oscillation probabilities, for an initially produced $\nu_{\alpha}$ to a finally detected $\nu_{\beta}$, can be written [26]

$$
P\left(\nu_{\alpha} \rightarrow \nu_{\beta}\right)=\delta_{\alpha \beta}-\sum_{j<k}\left[4 \operatorname{Re}\left(W_{\alpha \beta}^{j k}\right) \sin ^{2} \Delta_{k j}-2 \operatorname{Im}\left(W_{\alpha \beta}^{j k}\right) \sin 2 \Delta_{k j}\right]
$$

where

$$
W_{\alpha \beta}^{j k}=U_{\alpha j} U_{\alpha k}^{*} U_{\beta j}^{*} U_{\beta k},
$$

and $\Delta_{k j} \equiv 1.27 \delta m_{k j}^{2} L / E$. We assume that there are four mass eigenstates $m_{0}, m_{1}, m_{2}, m_{3}, m_{4}$, which are most closely associated with the flavor states $\nu_{s}, \nu_{e}, \nu_{\mu}$, and $\nu_{\tau}$, respectively. The solar oscillations are driven by $\delta m_{01}^{2}$, the atmospheric oscillations by $\delta m_{32}^{2}$, and the LSND oscillations by $\delta m_{02}^{2} \simeq$ $\delta m_{03}^{2} \simeq \delta m_{12}^{2} \simeq \delta m_{13}^{2}$. Hence, the solar oscillations are primarily $\nu_{e} \rightarrow \nu_{s}$ and the atmospheric oscillations are primarily $\nu_{\mu} \rightarrow \nu_{\tau}$. For oscillations of solar $\nu_{e}$ to sterile neutrinos, the solar data disfavors large mixing; hence the most likely solar solution is MSW small mixing. It is also possible to reverse the roles of $\nu_{s}$ and $\nu_{\tau}$; however, current data disfavor oscillations of atmospheric $\nu_{\mu}$ to sterile neutrinos [6].

The $4 \times 4$ mixing matrix $U$ may be parametrized by 6 mixing angles $\left(\theta_{01}\right.$, $\left.\theta_{02}, \theta_{03}, \theta_{12}, \theta_{13}, \theta_{23}\right)$ and 6 phases $\left(\delta_{01}, \delta_{02}, \delta_{03}, \delta_{12}, \delta_{13}, \delta_{23}\right)$; only three of the phases are observable in neutrino oscillations. A complete analysis of the four-neutrino mixing matrix is quite complicated. However, the smallness of the mixing indicated by the LSND results suggests that $\nu_{e}$ does not mix strongly with the two heavier states, i.e., that $\theta_{12}$ and $\theta_{13}$ are small. Similarly, one can assume that the other light state does not mix strongly with the heavier states, i.e., $\theta_{02}$ and $\theta_{03}$ are also small. This situation occurs naturally 
in the explicit four-neutrino models in the literature. Then after dropping terms second order in small mixing angles, $U$ takes the form [26]

$$
U=\left(\begin{array}{cccc}
c_{01} & s_{01}^{*} & s_{02}^{*} & s_{03}^{*} \\
-s_{01} & c_{01} & s_{12}^{*} & s_{13}^{*} \\
-c_{01}\left(s_{23}^{*} s_{03}+c_{23} s_{02}\right) & -s_{01}^{*}\left(s_{23}^{*} s_{03}+c_{23} s_{02}\right) & c_{23} & s_{23}^{*} \\
+s_{01}\left(s_{23}^{*} s_{13}+c_{23} s_{12}\right) & -c_{01}\left(s_{23}^{*} s_{13}+c_{23} s_{12}\right) & & \\
c_{01}\left(s_{23} s_{02}-c_{23} s_{03}\right) & s_{01}^{*}\left(s_{23} s_{02} c_{23} s_{03}\right) & -s_{23} & c_{23} \\
-s_{01}\left(s_{23} s_{12}-c_{23} s_{13}\right)+c_{01}\left(s_{23} s_{12}-c_{23} s_{13}\right) &
\end{array}\right),
$$

where $c_{j k} \equiv \cos \theta_{j k}$ and $s_{j k} \equiv \sin \theta_{j k} e^{i \delta j k}$. We see that under these conditions, $U$ has approximately block diagonal form. The mixing of solar neutrinos is due to $\theta_{01}$ and the mixing of atmospheric neutrinos is due to $\theta_{23}$; the values for these mixing angles are essentially given by the two-neutrino fits in Sec. 2 . Both vacuum and MSW solar oscillation solutions are allowed; for MSW oscillations to occur in the sun requires $m_{0}>m_{1}$. The mixing that drives the LSND oscillations is due to $\theta_{12}$ and $\theta_{13}$; the effective amplitude of the $\nu_{\mu} \rightarrow \nu_{e}$ oscillations in LSND is

$$
\sin ^{2} 2 \theta_{L S N D} \simeq 4\left|s_{12} c_{23}+s_{13} s_{23}^{*}\right|^{2} .
$$

The assumption that $\theta_{02}$ and $\theta_{03}$ are small is not required by current data. However, most explicit models have the approximate form given by Eq. (27); see Sec. 4.2. If in fact $\theta_{02}$ and $\theta_{03}$ are not small, $\nu_{e}$ oscillations to the flavor associated with the other light state $\left(\nu_{s}\right.$ or $\left.\nu_{\tau}\right)$ are possible at the LSND $L / E$ scale with an amplitude of the same order as the LSND oscillation amplitude.

\section{Consequences for Masses and Mixings}

\subsection{Three-neutrino models}

The atmospheric and solar data put restrictions on the neutrino mixing angles and mass-squared differences, but do not at all constrain the absolute neutrino mass scale, which must be determined by other methods. The freedom to choose the mass scale allows a wide variety of possible mass matrix structures, even for the same mass-squared differences and mixing.

The matrix $U$ that relates neutrino flavor eigenstates to neutrino mass eigenstates depends in general on mixing in both the neutrino and charged lepton sector. If $U_{\ell}$ diagonalizes the charged lepton mass matrix and $U_{\nu}$ the neutrino mass matrix, then $U=U_{\ell}^{\dagger} U_{\nu}$. Except where stated otherwise, in our discussions here we will work in the basis where the charged lepton mass matrix is diagonal, so that $U=U_{\nu}$. In general, the neutrino mass matrix 
in the flavor basis can then be written $M_{\alpha \beta}=\sum_{j} U_{\alpha j} m_{j} U_{\beta j}$ for Majorana neutrinos or $M_{\alpha \beta}=\sum_{j} U_{\alpha j} m_{j} U_{\beta j}^{*}$ for Dirac neutrinos (these are the same if $C P$ is conserved, i.e., when $U$ is real).

As an example of the different mass matrix structures that are possible, we consider the case when at least one of the masses is much smaller than the largest mass. Then there is one type of mass matrix of the form $M=$ $M_{0}+O\left(\delta m_{j k}^{2}\right)$ (up to trivial sign changes) that can lead to maximal mixing of atmospheric neutrinos:

$$
M_{0}=\frac{m}{2}\left(\begin{array}{lll}
0 & 0 & 0 \\
0 & a & b \\
0 & b & a
\end{array}\right)
$$

where $a, b \sim 1$. If $a=b$, then there is only one large mass $\left(m_{1}, m_{2} \ll m_{3} \equiv m\right)$ and the form of Eq. (29) automatically fixes $\theta_{23} \simeq \pi / 4$ and $\theta_{13} \simeq 0$; the $O\left(\delta m_{j k}^{2}\right)$ terms determine $\theta_{12}$. Bimaximal mixing $\left(\theta_{12} \simeq \pi / 4\right)$ is obtained if the leading $O\left(\delta m_{j k}^{2}\right)$ terms have the form

$$
\Delta M=\epsilon\left(\begin{array}{ccc}
0 & -1 & 1 \\
-1 & 0 & 0 \\
1 & 0 & 0
\end{array}\right)
$$

where $\epsilon \ll m$; subleading $O\left(\delta m_{j k}^{2}\right)$ terms are then needed to split $m_{1}$ and $m_{2}$. If $a \neq b$ in Eq. (29), then there are two large masses with $\theta_{23} \simeq \pi / 4$ and $\theta_{13} \simeq 0$. Small $\theta_{12}$, appropriate for the small-angle MSW solar solution, is achieved if the leading $O\left(\delta m_{j k}^{2}\right)$ terms then have the form

$$
\Delta M=\epsilon\left(\begin{array}{lll}
0 & 1 & 1 \\
1 & 0 & 0 \\
1 & 0 & 0
\end{array}\right)
$$

see Ref. 35] for an example of a GUT model that has this form.

In the situation where all masses are approximately degenerate, $m \equiv$ $\left|m_{1}\right| \simeq\left|m_{2}\right| \simeq\left|m_{3}\right| \gg \delta m_{j k}^{2}$, there are three different types of mass matrices of the form $M=M_{0}+O\left(\delta m_{j k}^{2}\right)$ (up to trivial changes in sign) that can lead to bimaximal mixing, depending on the relative signs of the $m_{j}$ :

$$
M_{0}=m\left(\begin{array}{ccc}
0 & -\frac{1}{\sqrt{2}} & \frac{1}{\sqrt{2}} \\
-\frac{1}{\sqrt{2}} & \frac{1}{2} & \frac{1}{2} \\
\frac{1}{\sqrt{2}} & \frac{1}{2} & \frac{1}{2}
\end{array}\right), \text { or } m\left(\begin{array}{lll}
1 & 0 & 0 \\
0 & 0 & 1 \\
0 & 1 & 0
\end{array}\right), \text { or } m\left(\begin{array}{lll}
1 & 0 & 0 \\
0 & 1 & 0 \\
0 & 0 & 1
\end{array}\right) \text {. }
$$

The requirement from neutrinoless double beta decay that the $\nu_{e} \nu_{e}$ element of the neutrino mass matrix be small (described below) implies that only the first case is allowed for Majorana neutrinos. The form of $M_{0}$ gives three degenerate neutrinos of mass $m$ and fixes two combinations of mixing angles $\left(c_{13} s_{12} \simeq-1 / \sqrt{2}\right.$ and $\left.c_{23} c_{12}-s_{13} s_{23} s_{12} \simeq 1 / 2\right)$, while the remaining degree of freedom among the mixing angles and the neutrino mass splittings are 
determined by the $O\left(\delta m_{j k}^{2}\right)$ terms. If the leading $O\left(\delta m_{j k}^{2}\right)$ terms are proportional to the mass matrix in Eq. (29) with $a=b, m_{3}$ is split from $m_{1}$ and $m_{2}, \theta_{13} \simeq 0$ and bimaximal mixing is obtained. Subleading $O\left(\delta m_{j k}^{2}\right)$ terms then provide the splitting between $m_{1}$ and $m_{2}$.

A different mixing scheme occurs if the neutrino mass matrix is approximately proportional to unity and the charged lepton mass matrix is close to the so-called democratic form 36 .

$$
M_{\ell}=\frac{m_{\ell}}{3}\left(\begin{array}{lll}
1 & 1 & 1 \\
1 & 1 & 1 \\
1 & 1 & 1
\end{array}\right) ;
$$

then there is one large eigenvalue $m_{\ell} \simeq m_{\tau}$ and two constraints on the flavor mixing angles, $c_{13} c_{23} \simeq 1 / \sqrt{3}$ and $s_{23} s_{12}-s_{13} c_{23} c_{12} \simeq 1 / \sqrt{3}$. If a small perturbation is added to the lower right element of $M_{\ell}$, the muon gets mass and $\theta_{13}$ is constrained to be approximately zero; then there is maximal mixing in the solar sector $\left(\sin ^{2} 2 \theta_{12}=1\right)$ and nearly maximal mixing in the atmospheric sector $\left(\sin ^{2} 2 \theta_{23}=8 / 9\right)$ 36]. Additional perturbations to the diagonal elements of the charged lepton and neutrino mass matrices are then needed to give the electron-muon and neutrino mass splittings, respectively.

An $S O(10)$ SUSY GUT model with a minimal Higgs sector can provide large $\nu_{\mu}-\nu_{\tau}$ mixing for atmospheric neutrinos, and can accommodate either small or large mixing of solar neutrinos 37. . Other possible neutrino mass matrix textures are discussed in Refs. [38], [39] and [40].

Although neutrino oscillations are not sensitive to the overall neutrino mass scale, there are other processes that do depend on absolute masses. For example, studies of the tritium beta decay spectrum put an upper limit on the effective mass of the electron neutrino

$$
m_{\nu_{e}} \equiv \sum_{j}\left|U_{e j}\right|^{2} m_{j}
$$

of about $2.5 \mathrm{eV}$ 41; in a three-neutrino model this implies an upper limit of $2.5 \mathrm{eV}$ on the heaviest neutrino 42 .

The current limit on the magnitude of the $\nu_{e}-\nu_{e}$ element of the neutrino mass matrix for Majorana neutrinos from neutrinoless double beta $(0 \nu \beta \beta)$ decay [43] is of order $0.5 \mathrm{eV}$ [44], taking into account the imprecise knowledge of the nuclear matrix element and the sensitivity of a background fluctuation analysis 45 . For the three-neutrino model this implies

$$
\begin{aligned}
\left|M_{\nu_{e} \nu_{e}}\right| & =\left|\sum_{j} U_{e j} m_{j} U_{e j}\right| \\
& =\left|\left(c_{13} c_{12}\right)^{2} m_{1}+\left(c_{13} s_{12}\right)^{2} m_{2} e^{i \phi_{2}}+s_{13}^{2} m_{3} e^{i \phi_{3}}\right| \leq M_{\max }=0.2 \mathrm{eV},
\end{aligned}
$$

where $\phi_{2}$ and $\phi_{3}$ are extra phases present for Majorana neutrinos that are not observable in neutrino oscillations. For models with one large mass $m_{1}, m_{2} \ll$ 
$m_{3} \simeq \sqrt{\delta m_{a t m}^{2}} \simeq 0.05 \mathrm{eV}$, and Eq. (36) does not provide any additional constraint. However, if all three masses are nearly degenerate $\left(m_{1} \simeq m_{2} \simeq m_{3} \equiv\right.$ $m)$, the $0 \nu \beta \beta$ decay limit becomes $s_{12}^{2} \geq\left[1-2 s_{13}^{2}-\left(M_{\max } / m\right)\right] /\left(2 c_{13}^{2}\right)$, which in turn implies that the solar $\nu_{e} \rightarrow \nu_{e}$ oscillation amplitude (see Eq. (24)) has the constraint 46

$$
4 c_{13}^{4} s_{12}^{2} c_{12}^{2} \geq 1-\left(\frac{M_{\max }}{m}\right)^{2}-2 s_{13}^{2}\left(1+\frac{M_{\max }}{m}\right) .
$$

For any value of $m>M_{\max } /\left(1-2 s_{13}^{2}\right)$ there will be a lower limit on the size of the solar $\nu_{e} \rightarrow \nu_{e}$ oscillation amplitude; for example, given the current limit on $s_{13}$, the small-angle MSW solar solution is ruled out for nearly degenerate Majorana neutrinos if $m>0.25 \mathrm{eV}$ [46]. Large-angle MSW and vacuum solar solutions, which have large mixing, are still allowed; any solar solution with maximal mixing can never be excluded by this bound.

Neutrino mass also provides an ideal hot dark matter component 47]; the contribution of neutrinos to the mass density of the universe is given by $\Omega_{\nu}=\sum m_{\nu} /\left(h^{2} 93 \mathrm{eV}\right)$, where $h$ is the Hubble expansion parameter in units of $100 \mathrm{~km} / \mathrm{s} / \mathrm{Mpc} 48$. For $h=0.65$, the model with three nearly degenerate neutrinos has $\Omega_{\nu} \simeq 0.075(\mathrm{~m} / \mathrm{eV})$. In three-neutrino models with hierarchical masses, in which the largest mass is of order $\sqrt{\delta m_{\text {atm }}^{2}}, \Omega_{\nu}$ is much smaller, on the order of 0.001 . Future measurements of the hot dark matter component may be sensitive to $\sum m_{\nu}$ down to $0.4 \mathrm{eV} 49$.

\subsection{Four-neutrino models}

As described in Sec. 3.2, four-neutrino models must have the $2+2$ mass structure, i.e., two nearly-degenerate pairs separated from each other by the LSND scale. One possible class of mass matrices that can give this situation is

$$
M=m\left(\begin{array}{cccc}
\epsilon_{1} & \epsilon_{2} & 0 & 0 \\
\epsilon_{2} & 0 & 0 & \epsilon_{3} \\
0 & 0 & \epsilon_{4} & 1 \\
0 & \epsilon_{3} & 1 & \epsilon_{5}
\end{array}\right)
$$

presented in the $\left(\nu_{s}, \nu_{e}, \nu_{\mu}, \nu_{\tau}\right)$ basis (i.e., the basis in which the charged lepton mass matrix is diagonal).

When $\epsilon_{5}=\epsilon_{4}$, the mass matrix in Eq. (37) can accommodate any of the three solar solutions, depending on the hierarchy of the mass matrix elements 32 .

$$
\begin{gathered}
\text { SAM : } \quad \epsilon_{2} \ll \epsilon_{1}, \epsilon_{4} \ll \epsilon_{3} \ll 1, \\
\text { LAM : } \quad \epsilon_{1}, \epsilon_{2}, \epsilon_{4} \ll \epsilon_{3} \ll 1, \\
\text { VO : } \epsilon_{1} \ll \epsilon_{2} \ll \epsilon_{4} \ll \epsilon_{3} \ll 1 .
\end{gathered}
$$


In each case, the mass eigenvalues have the hierarchy $m_{1}<m_{0} \ll m_{2}, m_{3}$, as required for the MSW solution. The mass matrix contains five parameters, just enough to incorporate the required three mass-squared differences and the oscillation amplitudes for the solar and LSND neutrinos. The large amplitude for atmospheric oscillations does not require an additional parameter, since the mass matrix naturally gives nearly-maximal mixing of $\nu_{\mu}$ with $\nu_{\tau}$. For the VO case, $\epsilon_{1}$ does not contribute to the phenomenology and can be set to zero, so that there are only four parameters; the large amplitude for solar oscillations also occurs naturally in this case [32,50].

Another variant with five parameters is $\epsilon_{5}=-\epsilon_{4}$ and $\epsilon_{2} \ll \epsilon_{1} \ll \epsilon_{3} \ll$ $\epsilon_{4} \sim 1$ [51. In this case, $\epsilon_{3}$ determines both the amplitude of the LSND oscillations and causes the splitting between $m_{2}$ and $m_{3}$ that drives the atmospheric oscillations. Two testable consequences of this model are that $\delta m_{\text {atm }}^{2} \leq 1.3 \times 10^{-3} \mathrm{eV}^{2}$ and that there should be observable $\nu_{e} \rightarrow \nu_{\tau}$ oscillations in short-baseline experiments.

For both of the previous cases $\left(\epsilon_{5}=\epsilon_{4} \ll 1\right.$ and $\left.-\epsilon_{5}=\epsilon_{4} \sim 1\right)$, the heavier mass pair is much heavier than the lighter mass pair, i.e., $m_{1}<m_{0} \ll m_{2}, m_{3}$. Yet another possibility is to have $\epsilon_{1}=\epsilon_{5}=0$ and $\epsilon_{3}, \epsilon_{4} \ll \epsilon_{2}<1$, where $\epsilon_{2}$ is not small compared to unity [50]. In this case, the two degenerate pairs of masses have mass eigenvalues that are the same order of magnitude; there are only four parameters as both the solar and atmospheric mixings are naturally close to maximal. However, the $m_{0}-m_{1}$ splitting in this model can give only MSW solar oscillations, which for large mixing are disfavored when $\nu_{e} \rightarrow \nu_{s}$.

Other four-neutrino mass matrix ansatzes have been presented in the literature [52], but they generally have characteristics similar to those discussed here. In all of the explicit four-neutrino models discussed above, since the $\nu_{e^{-}}$ $\nu_{e}$ element of the neutrino mass matrix vanishes, there is no contribution to neutrinoless double beta decay. However, because $m_{3}$ and $m_{4}$ are always of order $1 \mathrm{eV}$ or more (to provide the necessary mass-squared splitting for LSND oscillations), these models always contribute to the hot dark matter in the universe 53.

\section{$5 \quad$ Long-baseline experiments}

Long-baseline experiments (with $L / E_{\nu} \sim 10^{2}-10^{3} \mathrm{~km} / \mathrm{GeV}$ ) are expected to confirm the $\nu_{\mu} \rightarrow \nu_{\mu}$ disappearance oscillations at the $\delta m_{\text {atm }}^{2}$ scale. The K2K experiment [54] from KEK to SuperK, with a baseline of $L \simeq 250 \mathrm{~km}$ and a mean neutrino energy of $\left\langle E_{\nu}\right\rangle \sim 1.4 \mathrm{GeV}$ is underway. The MINOS experiment from Fermilab to Soudan [55], with a longer baseline $L \simeq 730 \mathrm{~km}$ and higher mean energies $\left\langle E_{\nu}\right\rangle=3,6$ or $12 \mathrm{GeV}$, is under construction and the ICANOE[56] and OPERA[57 experiments, with similar baselines from CERN to Gran Sasso, have been approved. These experiments with dominant $\nu_{\mu}$ and $\bar{\nu}_{\mu}$ beams will securely establish the oscillation phenomena and may 
measure $\delta m_{\mathrm{atm}}^{2}$ to a precision of order $10 \%$ [58. They will also measure the dominant oscillation amplitude $\sin ^{2} 2 \theta_{23}$.

Further tests of the neutrino parameters will likely require higher intensity neutrino beams, and $\nu_{e}, \bar{\nu}_{e}$ beams as well as $\nu_{\mu}, \bar{\nu}_{\mu}$, such as those generated in a neutrino factory 27,59, 60, 61]. The $\nu_{e}, \bar{\nu}_{e}$ components of the beam allow one to search for $\nu_{e} \rightarrow \nu_{\mu}$ and $\bar{\nu}_{e} \rightarrow \bar{\nu}_{\mu}$ appearance, which will occur in the leading $\delta m_{\text {atm }}^{2}$ oscillation if $\sin ^{2} 2 \theta_{13} \neq 0$. Depending on the machine parameters, $\delta m_{\mathrm{atm}}^{2}$ and $\sin ^{2} 2 \theta_{23}$ can be measured to an accuracy of $1-2 \%$, and $\sin ^{2} 2 \theta_{13}$ can be tested down to 0.01 or below 61. If the baseline is long enough $(L \gtrsim 1000 \mathrm{~km})$, matter effects in the Earth will also play an important role in an appearance experiment: for $\delta m_{\mathrm{atm}}^{2}>0\left(\delta m_{\mathrm{atm}}^{2}<0\right)$, $\nu_{e} \rightarrow \nu_{\mu}$ oscillations are enhanced (suppressed) and $\bar{\nu}_{e} \rightarrow \bar{\nu}_{\mu}$ oscillations are suppressed (enhanced). Therefore by comparing $\nu_{e} \rightarrow \nu_{\mu}$ with $\bar{\nu}_{e} \rightarrow$ $\bar{\nu}_{\mu}$ oscillations it may be possible to determine the sign of $\delta m_{\text {atm }}^{2}$ [61]. The enhancement due to matter of either $\nu_{e} \rightarrow \nu_{\mu}$ or $\bar{\nu}_{e} \rightarrow \bar{\nu}_{\mu}$ may also improve the $\sin ^{2} 2 \theta_{13}$ sensitivity for appropriate choices of $L$ and $E_{\nu}$ [61]. In a fourneutrino model, both short-baseline experiments with $L / E_{\nu} \sim 1 \mathrm{~km} / \mathrm{GeV}$ (which probe $\delta m_{\mathrm{LSND}}^{2}$ ) and long-baseline experiments will be required to obtain maximal information on the neutrino mixing parameters [26].

$C P$-violating effects due to the phase $\delta$ only become appreciable at the subleading $\delta m^{2}$ scale, and only then if the mixing angles are large enough [62]. In a three-neutrino model with $\delta m_{\text {sun }}^{2}$ and $\delta m_{\text {atm }}^{2}, C P$ violation will be observable only for the large-angle MSW solar solution; a long-baseline experiment with a high-intensity neutrino beam from a neutrino factory may be able to give information on $\delta$ in this case [63]. In a four-neutrino model, potentially large $C P$-violating effects are possible at the $\delta m_{\text {atm }}^{2}$ scale [26, 64].

\section{$6 \quad$ Summary and outlook}

In a three-neutrino world, it may be possible to completely determine the neutrino mixing matrix and two independent mass-squared differences using existing and planned neutrino oscillation experiments. Future measurements of solar neutrinos should pin down the neutrino mass and mixing parameters $\delta m_{21}^{2}$ and $\sin ^{2} 2 \theta_{12}$ that are predominantly responsible for the solar neutrino deficit. Long-baseline experiments can provide a more precise determination of $\delta m_{32}^{2}$ and $\sin ^{2} 2 \theta_{23}$, which drive the atmospheric neutrino anomaly, measure the size of $\sin ^{2} 2 \theta_{13}$, and determine the sign of $\delta m_{32}^{2}$. If the solar solution is large-angle MSW, long-baseline experiments may also be sensitive to the $C P$-violating phase $\delta$. Future measurements of beta decay spectra, doublebeta decay and hot dark matter may then help determine the last remaining neutrino mass parameter, the absolute neutrino mass scale.

In a four-neutrino world, there are three additional mixing angles and three additional $C P$ phases. Since the extra neutrino is sterile, it may be difficult to determine some of the additional mixing angles, especially if they 
are small, as is the case in many existing models. Short baseline experiments that probe the $\delta m_{\mathrm{LSND}}^{2}$ oscillation will be helpful in making sense of the larger parameter space. Furthermore, in four-neutrino models $C P$ violation may become observable at the $\delta m_{\text {atm }}^{2}$ scale (rather than the $\delta m_{\text {sun }}^{2}$ scale, as is the case in three-neutrino models), and there will be a contribution to hot dark matter on the order of $\sum m_{\nu} \sim 1 \mathrm{eV}$.

\section{References}

1. Kamiokande collaboration, K.S. Hirata et al., Phys. Lett. B280, 146 (1992); Y. Fukuda et al., Phys. Lett. B335, 237 (1994); IMB collaboration, R. BeckerSzendy et al., Nucl. Phys. Proc. Suppl. 38 B, 331 (1995); Soudan-2 collaboration, W.W.M. Allison et al., Phys. Lett. B391, 491 (1997); MACRO collaboration, M. Ambrosio et al., Phys. Lett. B434, 451 (1998).

2. J.G. Learned, S. Pakvasa, and T.J. Weiler, Phys. Lett. B207, 79 (1988); V. Barger and K. Whisnant, Phys. Lett. B209, 365 (1988); K. Hidaka, M. Honda, and S. Midorikawa, Phys. Rev. Lett. 61, 1537 (1988).

3. Super-Kamiokande Collaboration, Y. Fukuda et al., Phys. Rev. Lett. 81, 1562 (1998); Phys. Rev. Lett. 82, 2644 (1999).

4. G. Barr, T.K. Gaisser, and T. Stanev, Phys. Rev. D39, 3532 (1989); M. Honda, T. Kajita, K. Kasahara, and S. Midorikawa, Phys. Rev. D52, 4985 (1995); V. Agrawal, T.K. Gaisser, P. Lipari, and T. Stanev, Phys. Rev. D53, 1314 (1996); T.K. Gaisser et al., Phys. Rev. D54, 5578 (1996); T.K. Gaisser and T. Stanev, Phys. Rev. D57, 1977 (1998).

5. CHOOZ collaboration, M. Apollonio et al., Phys. Lett. B420, 397 (1998).

6. T. Kajita, talk presented at the 7th International Symposium on Particles, Strings and Cosmology (PASCOS 99), Granlibakken, CA, Dec. 1999, http://pc90.ucdavis.edu/talks/plenary/Kajita/

7. J.G. Learned, chapter in this book.

8. B.T. Cleveland et al., Nucl. Phys. B (Proc. Suppl.) 38, 47 (1995).

9. GALLEX Collaboration, W. Hampel et al., Phys. Lett. B388, 384 (1996); SAGE collaboration, J.N. Abdurashitov et al., Phys. Rev. Lett. 77, 4708 (1996).

10. Kamiokande collaboration, Y. Fukuda et al., Phys. Rev. Lett, 77, 1683 (1996);

11. Super-Kamiokande Collaboration, Y. Fukuda et al., Phys. Rev. Lett. 82, 1810 (1999); Phys. Rev. Lett. 82, 2430 (1999); M.B. Smy, hep-ex/9903034, to be published in Proc. of the APS Meeting of the Division of Particles and Fields (DPF-99), Los Angeles, California, January 1999; G. Sullivan, talk at the Aspen Winter Conference, January, 1999.

12. J.N. Bahcall and M.H. Pinsonneault, Rev. Mod. Phys. 67, 781 (1995); J.N. Bahcall, S. Basu, and M.H. Pinsonneault, Phys. Lett. B433, 1 (1998).

13. V. Barger, R.J.N. Phillips, and K. Whisnant, Phys. Rev. D24, 538 (1981); S.L. Glashow and L.M. Krauss, Phys. Lett. B190, 199 (1987); V. Barger, R.J.N. Phillips, and K. Whisnant, Phys. Rev. Lett. 65, 3084 (1990); ibid. 69, 3135 (1992); A. Acker, S. Pakvasa, and J. Pantaleone, Phys. Rev. D43, 1754 (1991); P.I. Krastev and S.T. Petcov, Phys. Lett. B285, 85 (1992); Phys. Rev. D53, 1665 (1996); N. Hata and P. Langacker, Phys. Rev. D56, 6107 (1997); S.L. Glashow, P.J. Kernan, and L.M. Krauss, Phys. Lett. B445, 412 (1999).

14. V. Barger and K. Whisnant, Phys. Rev. D59, 093007 (1999). 
15. J.N. Bahcall, P.I. Krastev, and A.Yu. Smirnov, Phys. Rev. D58, 096016 (1998).

16. L. Wolfenstein, Phys. Rev. D17, 2369 (1978); V. Barger, R.J.N. Phillips, and K. Whisnant, Phys. Rev. D22, 2718 (1980); P. Langacker, J.P. Leveille, and J. Sheiman, Phys. Rev. D27, 1228 (1983); S.P. Mikheyev and A. Smirnov, Yad. Fiz. 42, 1441 (1985); Nuovo Cim. C9, 17 (1986); S.J. Parke, Phys. Rev. Lett. 57, 1275 (1986); S.J. Parke and T.P. Walker, Phys. Rev. Lett. 57, 2322 (1986); W.C. Haxton, Phys. Rev. Lett. 57, 1271 (1986).

17. A. McDonald, Solar Neutrino Observatory (SNO) collaboration, talk at Neutrino-98, Takayama, Japan, June 1998.

18. L. Oberauer, Borexino collaboration, talk at Neutrino-98, Takayama, Japan, June 1998.

19. F. Arneodo, ICARUS collaboration, Nucl. Phys. Proc. Suppl. 70, 453 (1999).

20. V. Barger, R.J.N. Phillips, and K. Whisnant, Phys. Rev. Lett. 65, 3084 (1990); ibid. 69, 3135 (1992); S. Pakvasa and J. Pantaleone, Phys. Rev. Lett. 65, 2479 (1990); A. Acker, S. Pakvasa, and J. Pantaleone, Phys. Rev. D43, 1754 (1991); P.I. Krastev and S.T. Petcov, Phys. Lett. B285, 85 (1992); Nucl. Phys. B449, 605 (1995); S.P. Mikheyev and A.Yu. Smirnov, Phys. Lett. B429, 343 (1998); B. Faid, G.L. Fogli, E. Lisi, and D. Montanino, Astropart. Phys. 10, 93 (1999); S.L. Glashow, P.J. Kernan, and L.M. Krauss, Phys. Lett. B445, 412 (1999); J.M. Gelb and S.P. Rosen, Phys. Rev. D60, 011301 (1999); V. Berezinsky, G. Fiorentini, and M. Lissia, hep-ph/9811352; V. Barger and K. Whisnant, Phys. Lett. B456, 54 (1999).

21. Liquid Scintillator Neutrino Detector (LSND) collaboration, C. Athanassopoulos et al., Phys. Rev. Lett. 75, 2650 (1995); 77, 3082 (1996); Phys. Rev. C58, 2489 (1998).

22. D.O. Caldwell, G.M. Fuller, and Y.-Z. Qian, Phys. Rev. D61, 123005 (2000).

23. E. Church et al. (BooNE Collab.), "A letter of intent for an experiment to measure $\nu_{\mu} \rightarrow \nu_{e}$ oscillations and $\nu_{\mu}$ at the Fermilab Booster", May 16, 1997, unpublished.

24. V. Barger, T.J. Weiler, and K. Whisnant, Phys. Lett. B440, 1 (1998).

25. L.-L. Chau and W.-Y. Keung, Phys. Rev. Lett. 53, 1802 (1984); C. Jarlskog, Z. Phys. C29, 491 (1985); Phys. Rev. D35, 1685 (1987).

26. V. Barger, Y.-B. Dai, K. Whisnant, and B.-.L. Young, Phys. Rev. D59, 113010 (1999).

27. A. De Rujula, M.B. Gavela, and P. Hernandez, Nucl. Phys. B547, 21 (1999).

28. V. Barger, K. Whisnant, R.J.N. Phillips, Phys. Rev. Lett. 45, 2084 (1980); S. Pakvasa, in Proc. of the XXth International Conference on High Energy Physics, ed. by L. Durand and L.G. Pondrom, AIP Conf. Proc. No. 68 (AIP, New York, 1981), Vol. 2, p. 1164; D.J. Wagner and T.J. Weiler, Phys. Rev. D59, 113007 (1999); A.M. Gago, V. Pleitez, R.Z. Funchal, Phys. Rev. D61, 016004 (2000); K.R. Schubert, hep-ph/9902215; K. Dick, M. Freund, M. Lindner and A. Romanino, Nucl. Phys. B562, 29 (1999); J. Bernabeu, hep-ph/9904474; S.M. Bilenky, C. Giunti, and W. Grimus, Phys. Rev. D58, 033001 (1998); M. Tanimoto, Prog. Theor. Phys. 97, 901 (1997); J. Arafune, J. Sato, Phys. Rev. D55, 1653 (1997); T. Fukuyama, K. Matasuda, H. Nishiura, Phys. Rev. D57, 5844 (1998); M. Koike and J. Sato, hep-ph/9707203, Proc. of the KEK Meetings on CP Violation and its Origin; H. Minakata and H. Nunokawa, Phys. Lett. B413, 369 (1997); H. Minakata and H. Nunokawa, Phys. Rev. D57, 4403 (1998); J. Arafune, M. Koike and J. Sato, Phys. Rev. D56, 3093 
(1997); M. Tanimoto, Phys. Lett. B435, 373 (1998); H. Fritzsch and Z.Z. Xing, Phys. Rev. D61, 073016 (2000); Z.Z. Xing, hep-ph/0002246.

29. V. Barger, S. Pakvasa, T.J. Weiler, and K. Whisnant, Phys. Lett. B437, 107 (1998); A.J. Baltz, A. Goldhaber, and M. Goldhaber, Phys. Rev. Lett. 81, 5730 (1998); F. Vissani, hep-ph/9708483; M. Jezabek and Y. Sumino, Phys. Lett. B440, 327 (1998); Y. Nomura and T. Yanagida, Phys. Rev. D59, 017303 (1999); G. Altarelli and F. Feruglio, Phys. Lett. B439, 112 (1998); JHEP 11, 021 (1998); H. Georgi and S. Glashow, Phys. Rev. D61, 097301 (2000); S. Davidson and S.F. King, Phys. Lett. B445, 191 (1998); R.N. Mohapatra and S. Nussinov, Phys. Lett. B441, 299 (1998); Phys. Rev. D60, 013002 (1999). S.K. Kang and C.S. Kim, Phys. Rev. D59, 091302 (1999); C. Jarlskog, M. Matsuda, S. Skadhauge, and M. Tanimoto, Phys. Lett. B449, 240 (1999); E. Ma, Phys. Lett. B456, 48 (1999). D.V. Ahluwalia, Mod. Phys. Lett. A13, 2249 (1998); I. Stancu and D.V. Ahluwalia, Phys. Lett. B460, 431 (1999).

30. LEP Electroweak Working Group and SLD Heavy Flavor Group, D. Abbaneo et al., CERN-PPE-96-183, December 1996.

31. S.M. Bilenky, C. Giunti, and W. Grimus, Eur. Phys. J. C1, 247 (1998).

32. V. Barger, S. Pakvasa, T.J. Weiler, and K. Whisnant, Phys. Rev. D58, 093016 (1998).

33. Y. Declais et al., Nucl. Phys. B434, 503 (1995).

34. F. Dydak et al., Phys. Lett. B134, 281 (1984).

35. J.K. Elwood, N. Irges, and P. Ramond, Phys. Rev. Lett. 81, 5064 (1998).

36. H. Fritzsch and Z. Xing, Phys. Lett. B372, 265 (1996); 440, 313 (1998); E. Torrente-Lujan, Phys. Lett. B389, 557 (1996); M. Fukugita, M. Tanimoto, and T. Yanagida, Phys. Rev. D57, 4429 (1998); M. Tanimoto, Phys. Rev. D59, 017304 (1999).

37. C.H. Albright and S.M. Barr, Phys. Rev. D58, 013002 (1998); Phys. Lett. B461, 218 (1999).

38. R. Barbieri, L.J. Hall, and A. Strumia, Phys. Lett. B445, 407 (1999); L.J. Hall and D. Smith, Phys. Rev. D59, 113013 (1999).

39. R.N. Mohapatra, chapter in this book; G. Altarelli and F. Feruglio, Phys. Rep. 320, 295 (1999); P. Ramond, hep-ph/0001009, in Proc. of the 6th Int'l. Workshop on Topics in Astroparticle and Underground Physics (TAUP 99), Paris, Sept. 1999; S.M. Barr and I. Dorsner, hep-ph/0003058; and references therein.

40. S.F. King, Phys. Lett. B439, 350 (1998); Nucl. Phys. 562, 57 (1999); C.H. Albright and S.M. Barr, Phys. Rev. D58, 013002 (1998).

41. A.I. Belesev et al., Phys. Lett. B350, 263 (1995); V.M. Lobashev et al., Phys. Lett. B460, 227 (1999).

42. V. Barger, T.J. Weiler, and K. Whisnant, Phys. Lett. B442, 255 (1998).

43. M. Doi et al., Phys. Lett. B102, 323 (1981); H. Minakata and O. Yasuda, Phys. Rev. D 56, 1692 (1997); H. Georgi and S.L. Glashow, Phys. Rev. D61, 097301 (2000); J. Ellis and S. Lola, Phys. Lett. B458, 310 (1999); F. Vissani, JHEP 9906, 022 (1999); S.M. Bilenki, C. Giunti, W. Grimus, B. Kayser, and S.T. Petcov, Phys. Lett. B465, 193 (1999).

44. L. Baudis et al., Phys. Rev. Lett. 83, 41 (1999).

45. D. Caldwell (private communication).

46. V. Barger and K. Whisnant, Phys. Lett. B456, 194 (1999).

47. J.R. Primack, Science 280, 1398 (1998); E. Gawiser and J. Silk, Science 280, 1405; J.R. Primack and M.A.K. Gross, astro-ph/9810204, in Proc. of the 10th Rencontres de Blois: The Birth of Galaxies, Blois, France, June 1998. 
48. E.W. Kolb and M.S. Turner, The Early Universe (Addison-Wesley, 1990).

49. D.J. Eisenstein, W. Hu, and M. Tegmark, Phys. Rev. Lett. 80, 5255 (1998); astro-ph/9807130.

50. S. Mohanty, D.P. Roy, and U. Sarkar, Phys. Lett. B445, 185 (1998).

51. S.C. Gibbons, R.N. Mohapatra, S. Nandi, and A. Raychaudhuri, Phys. Lett. B430, 296 (1998).

52. D.O. Caldwell and R.N. Mohapatra, Phys. Rev. D48, 3259 (1993); J.T. Peltoniemi and J.W.F. Valle, Nucl. Phys. B406, 409 (1993); R. Foot and R.R. Volkas, Phys. Rev. D52, 6595 (1995); Ernest Ma and Probir Roy, Phys. Rev. D52, R4780 (1995); J.J. Gomez-Cadenas and M.C. Gonzalez-Garcia, Z. Phys. C71, 443 (1996); N. Okada and O. Yasuda, Int. J. Mod. Phys. A12, 3669 (1997); R.N. Mohapatra, hep-ph/9711444, in Proc. of the Workshop on Physics at the First Muon Collider and at the Front End of a Muon Collider, Fermilab, Nov. 1997, ed. by S. Geer and R. Raja (AIP, Woodbury, 1998), AIP Conf. Proc. 435, p. 358; N. Gaur, A. Ghosal, Ernest Ma, and Probir Roy, Phys. Rev. D58, 071301 (1998).

53. D.O. Caldwell and J.R. Primack, Phys. Rev. Lett. 74, 2160 (1995).

54. K. Nishikawa et al. (KEK-PS E362 Collab.), "Proposal for a Long Baseline Neutrino Oscillation Experiment, using KEK-PS and Super-Kamiokande", 1995, unpublished; INS-924, April 1992, submitted to J. Phys. Soc. Jap.; Y. Oyama, Proc. of the YITP Workshop on Flavor Physics, Kyoto, Japan, 1998, hepex/9803014.

55. MINOS Collaboration, "Neutrino Oscillation Physics at Fermilab: The NuMIMINOS Project," NuMI-L-375, May 1998.

56. See the ICANOE web page at http://pcnometh4.cern.ch

57. See the OPERA web page at http://www.cern.ch/opera/

58. D. A. Petyt, "A study of parameter measurement in a long-baseline neutrino oscillation experiment", thesis submitted to Univ. of Oxford, England, 1998.

59. S. Geer, "Neutrino beams from muon storage rings: characteristics and physics potential", FERMILAB-PUB-97-389, 1997, presented at the Workshop on Physics at the First Muon Collider and Front-End of a Muon Collider, November, 1997; S. Geer, Phys. Rev. D57, 6989 (1998); C. Ankenbrandt et al. (Muon Collider Collaboration), Phys. Rev. ST Accel. Beams 2, 081001 (1999); R.B. Palmer et al. (Muon Collider Collababoration), http://pubweb/bnl.gov/people/palmer/nu/params.ps

60. M. Campanelli, A. Bueno, and A. Rubbia, hep-ph/9905240; I. Mocioiu and R. Shrock, hep-ph/9910554, hep-ph/0002149; A. Cervera et al., hep$\mathrm{ph} / 0002108$.

61. V. Barger, S. Geer, and K. Whisnant, Phys. Rev. D61, 053004 (2000); V. Barger, S. Geer, R. Raja, and K. Whisnant, Phys. Rev. D62, 013004 (2000).

62. This was first discussed in the context of three neutrinos in V. Barger, K. Whisnant, and R.J.N. Phillips, Phys. Rev. Lett. 45, 2084 (1980).

63. M. Freund, M. Lindner, S.T. Petcov, and A. Romanino, hep-ph/9912457; V. Barger, S. Geer, R. Raja, and K. Whisnant, hep-ph/0003184, Phys. Rev. D (in press).

64. S.M. Bilenky, C. Giunti, and W. Grimus, Phys. Rev. D58, 033001 (1998); Prog. Part. Nucl. Phys. 43, 1 (1999); T. Hattori, T. Hasuike, and S. Wakaizumi, hep$\mathrm{ph} / 0002096$. 